\title{
OPTICAL COHERENCE TOMOGRAPHY ANGIOGRAPHY TO DETECT NON-EXUDATIVE NEOVASCULAR AGE-RELATED MACULAR DEGENERATION MIMICKING CHRONIC CENTRAL SEROUS CHORIORETINOPATHY
}

\author{
${ }^{1}$ Manuel A P Vilela, \\ ${ }^{1}$ Federal University of Health Sciences of Porto Alegre, RS, Brazil.
}

\begin{abstract}
Chronic central serous chorioretinopathy (CCSC) can produce an overlap of signs that can be difficult to distinguish from cases with non-exudative neovascular age-related macular degeneration (nneAMD). Around 2-10\% of the patients with cCSC will develop neovascularization. Sometimes both diseases could be present at the same individual. The purpose of this narrative review is to discuss the current information concerning the signs in OCT angiography (OCTA) that eventually could help to differentiate these situations and analyze the best diagnostic evidence.
\end{abstract}

Keywords: Central serous chorioretinopathy, Optical coherence tomography angiography, age-related macular degeneration, macula, retina

Cite This Article: VILELA, manuel a p. Optical Coherence Tomography Angiography to detect non-exudative neovascular age-related macular degeneration mimicking chronic central serous chorioretinopathy.. International Journal of Retina, [S.I.], v. 3, n. 2, sep. 2020. ISSN 2614-8536. Available at: https://www.ijretina.com/index.php/ijretina/article/view/104

*Correspondence to: Manuel A P Vilela, Federal University of Health Sciences of Porto Alegre, RS, Brazil,

mapvilela@gmail.com

\section{INTRODUCTION}

Most of the time it is not hard to differentiate chronic central serous chorioretinopathy (cCSC) from non-exudative neovascular agerelated macular degeneration (nneAMD). But in older patients (60-70 years old) with chronic subretinal/intraretinal fluid (SRF/IRF) and retinal pigment epithelium (RPE)/Bruch's membrane (BM) changes might produce an area of overlap that can be difficult to distinguish. And eventually, both diseases could be present at the same individual. These are situations with some similarities even for the commonest diagnostic technologies used in real-life practice like optical coherence tomography (OCT), intravenous fluorescein angiography (IVFA), or indocyanine green angiography (ICGA). The correct recognition is of pivotal importance cause it could be related to the potential presence of choroidal neovascularization (CNV) - especially type 1 and severe functional visual loss. For the CSC the prognosis is much better (all in all $80 \%$ will retain $20 / 40$ or more). However, $12 \%$ of these patients can have a bad prognosis, with legally blind in both eyes after 10 years. CNV has been reported in $2-10 \%$ of the CSC cases. OCT B scan shows specific signals like drusen in AMD, but SRF/IRF, pigment epithelium detachment (PED) and modifications at the outer retina and RPE occurs in both situation. In chronic cases, some of these signals should be valorized. ${ }^{1-3}$ 
The purpose is to review the current information concerning the signs in OCT angiography (OCTA) that eventually could help to differentiate these situations and analyze the best diagnostic evidence.

\section{CLINICAL DETECTION ASPECTS}

Dye-based angiographies can localize on CSC - but not in all cases - the leakage site at the RPE plane. It could be uni or multifocal, uni or bilateral. The leakage and the height of the subretinal fluid (SRF) or another sign reduces the conditions to find out active or chronic choroidal neovascularization. ${ }^{4-6}$ Table summarizes how to make a precise diagnosis with the use of dye-based angiographies and B-scan OCT.

Double sign and time for development are strongly related to CNV in both diseases, and especially the time for developing CNV is longer in both situations (more than 10 years in CSC). ${ }^{1,7}$ It is in the cCSC where some aspects are shared, sometimes inconclusive (basically in the absence of the descend traits) with nneAMD including (a) irregular and detached RPE; (b) thicker, opaque or hyperdense SRF or IRF (CME pattern), (c) MLE/EZ modifications, (d) foveal thinning and (e) increased choroidal thickness. Sometimes the pachychoroid spectrum is seen in both cases ${ }^{8}$.

The recent use of OCTA has shown identical efficiency than the gold standard diagnostic resources, with high sensitivity and specificity. Sometimes it appears better than the gold standard but other times a little bit worst. Considering the facilities it tends to be more used. ${ }^{1-8}$

In different studies, the prevalence range of CNV diagnosis using OCTA in CCSC is $8.3-44.8 \%$. This heterogeneity is too high. There are significant differences between these populations (sample size, different inclusion/exclusion criteria, cross-sectional or longitudinal study). Such variability was assumed to be not only due to sampling errors but also to the different effects on the population. In real-life, these results are much better than isolated IVFA, and more specific than the combination of IVFA/OCT. ${ }^{8-14}$

Table 1. Diagnostic Aspects of Csc and Amd

CSC

More likely to be smaller, circular, regular, and with clear content (81\%). If Irregular, or flat, dense (chronic) $=>$ CNV
AMD

\begin{tabular}{ll}
\hline PED & More likely to be smaller, circular, \\
& regular, and with clear content \\
& (81\%). If Irregular, or flat, dense \\
& (chronic) $=>$ CNV
\end{tabular}

Higher, irregular, thicker, multiple and with more discontinuities

\begin{tabular}{ll}
\hline SRF & $\begin{array}{l}\text { Higher and if IRF present (cystoid } \\
\text { edema) }=>\text { CNV }\end{array}$
\end{tabular}

\begin{tabular}{ll}
\hline EXTERNAL RETINA & Hypertrophic outer retinal changes \\
& are more common (but less \\
& expressive)
\end{tabular}

Hyper-reflective band (outer retina/RPE) with posterior shadowing (42.9\%);

MLE/EZ discontinuities more expressive and prevalent

CT Increased (not all cases) Pachy (42\%)


In OCTA during acute CSC we can identify high flow signals. Hyper/mixed choroidal perfusion pattern is present in almost $100 \%$ at this stage. In cCSC hyperperfusion is seen only in $50 \%$ and this pattern is definitively different from normal subjects. ${ }^{15,16}$ Chan et $\mathrm{al}^{5}$ showed that OCTA in CCSC with irregular PED have demonstrated dilated capillaries inside (21/26 patients $81 \%$ ) and not a suspect pattern in FA (78\% - 14/18 eyes). The presence of an irregular, flat, and dense PED in cCSC is a strong indication of the CNV existence. This specific situation shows more sensitivity of the OCTA (35.6\%) versus the combination of dye-based angiographies and structural B san OCT (25\%). ${ }^{4,12}$ When used for this specific differentiation the ICGA and OCTA seem to be quite similar and their diagnostic capability ranges from $68 \%$ $100 \% .{ }^{17}$ This evidence supports the use of the new method considering all the facilities involved. (Figure)
Other relevant signs in OCTA are the flow void zones and darker areas. Probably they are related to the choroidal perfusion state. First of all, they are frequent in both cases (AMD ou CSC). These patterns indicate a potential long-term contribution to pathogenesis. For CSC they are described in areas (75\%) and spots (52.9\%). The age and CCSC have shown an increased total area of flow voids. The presence of higher SRF ( $485 \mathrm{~nm}$ ) blocks the choriocapillaris (CC) analysis. And the state of the deeper choroidal vessels can be related to changes in RPE and retina. ${ }^{14-16,18,19}$ These flow void areas mean microcirculatory deficiencies are detected in the fellow eyes of both situations and this pattern is also different from normal subjects. This could explain (observing the unaffected eyes) how the long-standing dilated choroidal vessels can affect the CC/RPE. Recurrent episodes in CCSC have demonstrated abnormal flow even after resolution. 20,21

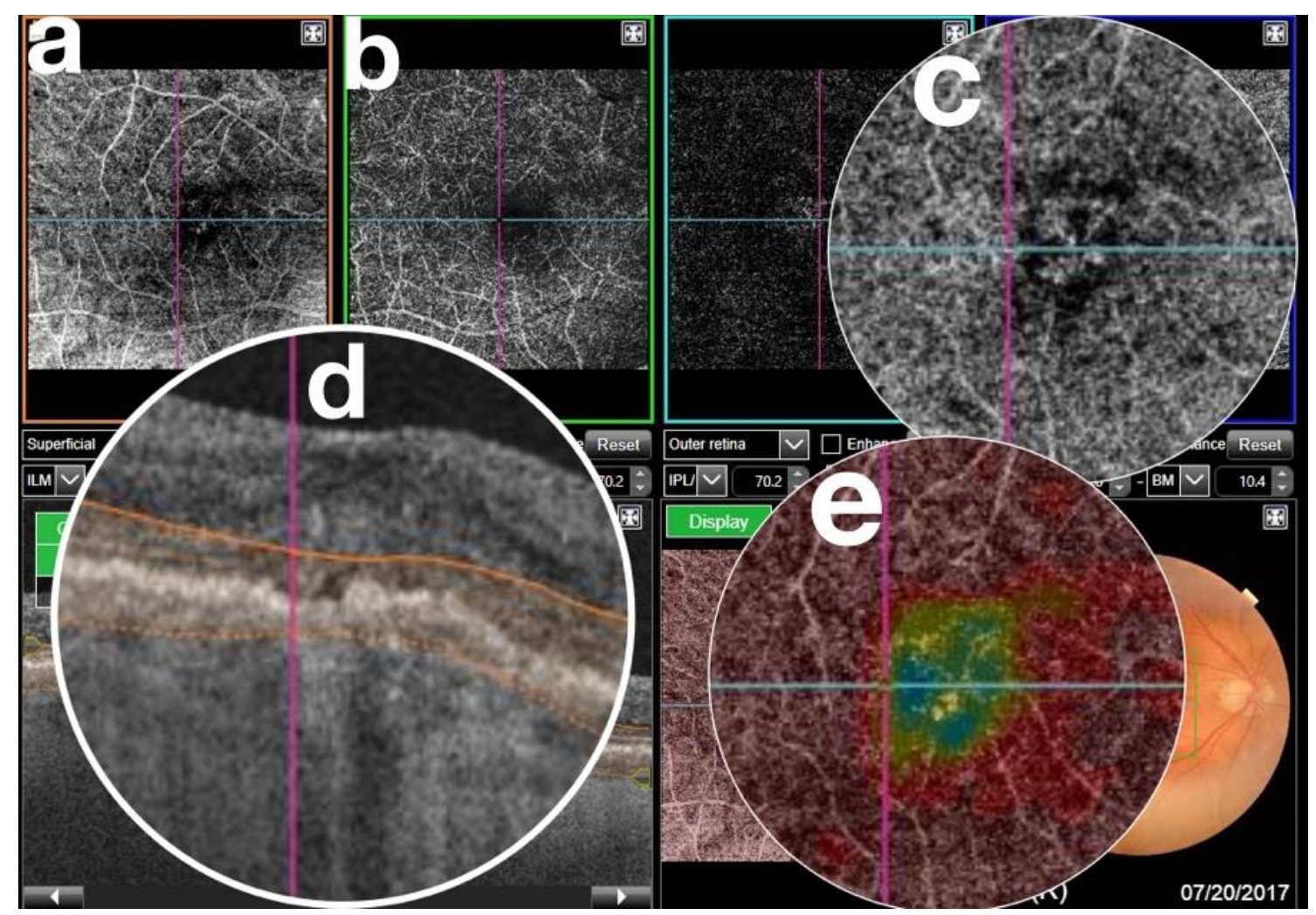

Figure 1. cCSC in 81years old male. Superficial (a) and deeper (b) plexus preserved but a CNV is evident (c ). Manual segmentation (d) helps the diagnosis (e)

In conclusion, nneAMD and cCSC sometimes have some similar aspects in older age, and because of that, the detection of a CNV is essential. OCTA can help in this differentiation with very good sensitivity in a rapid and noninvasive way. 


\section{REFERENCES}

1. Daruich A, Matet A, Dirani A, et al. Central serous chorioretinopathy: Recent findings and new physiopathology hypothesis. Prog Retin Eye Res 2015; 48:82-118.

2. Daruich $A$, Matet $A$, Behar-Cohen F. Central serous chorioretinopathy. In: Vilela MAP, Putz C, Dantas AM (eds). Retina Clínica. Rio de Janeiro, Cultura Médica, 2016.

3. Mrejen M, Balaratnasingam C, Kaden TR, et al. Longterm Visual Outcomes and Causes of Vision Loss in Chronic Central Serous Chorioretinopathy. Ophthalmology 2019; 126(4):576-588.

4. Hage R, Mrejen S, Krivosic V, et al. Flat irregular retinal pigment epithelium detachments in chronic central serous chorioretinopathy and choroidal neovascularization. Am J Ophthalmol 2015;159(5):890-903.

5. Chan SY, Wang Q, Wei WB, Jonas JB. Optical coherence tomographic angiography in central serous chorioretinopathy. Retina 2016;36:20512058.

6. Cheung $C M G$, Lee $W K$, Koizumi $H$, et al. Pachychoroid disease. Eye 2019; 33(1):14-33.

7. Fung AT, Yannuzzi LA, Freund KB. Type 1(sub-retinal pigment epithelial) neovascularization in central serous chorioretinopathy masquerading as neovascular age-related macular degeneration. Retina 2012;32:1829-1837.

8. Moussa M, Leila M, Khalid H, Lolah M. Detection of Silent Type I Choroidal Neovascular Membrane in Chronic Central Serous Chorioretinopathy Using En Face Swept-Source Optical Coherence Tomography Angiography J Ophthalmol 2017, Article ID 6913980, https://doi.org/10.1155/2017/6913980.

9. Bonini Filho $M A$, de Carlo $T E$, Ferrara $D$, et al. Association of Choroidal Neovascularization and Central Serous Chorioretinopathy With Optical Coherence Tomography Angiography. JAMA Ophthalmol 2015; 133(8):899-906.

10. Quaranta-El Maftouhi M, El Maftouhi A, Eandi CM. Chronic central serous chorioretinopathy imaged by optical coherence tomographic angiography. Am J Ophthalmol 2015;160(3):581-587.
11. Gołębiewska, J, Brydak-Godowska J, Moneta-Wielgos $\mathrm{J}$, et al. Correlation between Choroidal Neovascularization Shown by OCT Angiography and Choroidal Thickness in Patients with Chronic Central Serous Chorioretinopathy J Ophthalmol 2017;Article ID 3048013, https://doi.org/10.1155/2017/3048013.

12. Bosquet $E$, Bonnin $S$, Mrejen $S$, et al. Optical coherence tomography angiography of flat irregular pigment epithelium detachment in chronic central serous chorioretinopathy.. Retina 2018;38(3):629-638

13. Sahoo NK, Mishra SB, lovino C, et al. Optical coherence tomography angiography findings in cystoid macular degeneration associated with central serous chorioretinopathy.. Br J Ophthalmol 2019; doi: 10.1136/bjophthalmol-2018-313048.

14. Tang P, Shields R, Silva RA. Optical Coherence Tomography Angiography Findings in Chronic Central Serous Chorioretinopathy After Photodynamic Therapy. Ophthalmic Surg Lasers Imaging Retina 2019;50(1):25-32.

15. Seo EJ, Um T, Yoon YH. Abnormal choroidal flow on optical coherence tomography angiography in central serous chorioretinopathy. Clin Exp Ophthalmol 2019;47(4):505-512.

16. De Bats F, Cornut P-L, Wolff B et al. Dark and white lesions observed in central serous chorioretinopathy on optical coherence tomography angiography. Eur J Ophthalmol 2018;28(4):446-453.

17. Min JY, Lv Y, Yu S, Gong YY. Findings of OCTangiography compared to fluorescein and indocyanine green angiography in central serous chorioretinopathy. Lasers Surg Med 2018;50(10):987993.

18. Savastano MC, Dansingani KK, Rispoli $M$, et al. Classification of Haller vessel arrangements in acute and chronic central serous chorioretinopathy imaged with en face optical coherence tomography. Retina 2018;38(6):1211-1215.

19. Matet A, DAruich A, Hardy S, Behar-Cohen F. Patterns of choriocapillaris flow signal voids in central serous chorioretinopathy: an optical coherence tomography angiography study. Retina 2019; doi: 10.1097/IAE.0000000000002271 
20. Rochepeau C, Kodjikian L, Garcia MA, et al. Optical Coherence Tomography Angiography Quantitative Assessment of Choriocapillaris Blood Flow in Central Serous Chorioretinopathy. Am J Ophthalmol 2018;194:26-34.
21. Yun C, Huh J, Ahn SM, et al. Choriocapillaris flow features and choroidal vasculature in the fellow eyes of patients with acute central serous chorioretinopathy. Graefes Arch Clin Exp Ophthalmol 2019;257(1):57-70.

\section{@ $(\Theta \Theta$}

This work licensed under Creative Commons Attribution 